\title{
Doppler Echocardiographic Indices in Aortic Coarctation: A Comparison of Profiles before and After Stenting
} \author{
Allam $^{2}$, Sameh I Sersar ${ }^{5}$ and Mohamed Alassal ${ }^{4 *}$ \\ ${ }^{1}$ National Heart Institute, Egypt \\ ${ }^{2}$ Cardiology Department, Alazhar University, Egypt \\ ${ }^{3}$ Cardiothoracic Surgery Department, Alazhar University, Egypt \\ ${ }^{4}$ Cardiothoracic Surgery Department, Benha University, Egypt \\ ${ }^{5}$ Cardiothoracic Surgery Department, Mansoura University, Egypt
}

Diaa Mohamed Shalaby¹, Ahmed Moawed Alemam ${ }^{1}$, Abdelaziz rizk ${ }^{2}$, A Elsebaeyshousha ${ }^{3}$ and Sameh Refaat

Submission: May 25, 2018; Published: June 27, 2018

*Corresponding author: Mohamed Abdelwahab Alassal MD, Associate Professor and Consultant Cardiothoracic Surgeon, Benha Faculty of Medicine, Egypt, Email: dmohamedabdelwahab@gmail.com.

Abstract

Background: Aortic coarctation is one of the most common congenital heart defects. Nowadays, percutaneous stenting is an accepted form of treatment for isolated coarctation of the aorta by Balloon expandable endovascular stents, Diagnosis and evaluation of coarctation is of great importance, not only before stenting but also after implantation, in order to assess the success of procedure and occurrence of restenosis in followup. Cardiac magnetic resonance is the imaging modality of choice. However, it has limited availability or clinical contraindication. On the other hand, two-dimensional and Doppler echocardiographic techniques are simple, widely available, imaging tools which can be used for the indirect evaluation of coarctation.

Objectives: The aim of this work is to (1) evaluate the changes in a complete list of echocardiographic profiles in patients with aortic coarctation before and after stenting (2) and to determine the diagnostic value of these indices as an indicator of success of aortic coarctation stenting.

Patients and methods: This prospective study was conducted on 40 patients with a diagnosis of aortic coarctation based on angiography. Echocardiographic assessment was done twice for all patients before and after stenting, complete lists of Doppler indices were recorded for each case. After comparing the values of indices before and after stenting, diagnostic values of each index as indicator of success of procedure was statistically assessed.

Results: This study enrolled 40 patients, with diagnosed coarctation of aorta), 32 males and 8 females with a mean age of $15.14 \pm 9.17$ years (range 6.5-28) were enrolled in this study, the mean length of the stenosis was $18.5+\_9.5 \mathrm{~mm}$. In addition, the mean baseline ejection fraction was $58.55 \pm 5.10 \%$ (range $40-60 \%$ ) and 36 of patients were hypertensive. Except for the mean velocity and mean pressure gradient of the abdominal aorta, the values of the other indices of the abdominal/descending aorta showed significant change after stenting to indicate significant diagnostic accuracy for detecting success of stenting, and we recommend to use these indices as individualized reference for each case during follow up after stenting to detect incidence of restenosis. The, acceleration time, (m/s), Time to peak systolic velocity (m/s), velocity-time integral and the pressure half-time were among the indices with the highest accuracy rates for this purpose $(\mathrm{p}<0.001)$.

Conclusion: Post-stenting echocardiographic profiles could provide a reliable reference value of the normal aortic haemodynamics as a unique identification of each patient and it is presumed that these indices could be used as reliable indicators of response to treatment.

Keywords: Aortocaval fistula; Surgery; CT angiography

\section{Introduction}

As one of the most common congenital heart defects aortic coarctation [1] has a wide morphological spectrum that varies from transverse arch and isthmal hypoplasia, which are seen most

commonly in new-born babies, to discrete stenosis or membrane like obstructions, which are typically observed in older patients [2]. 
Aortic coarctation presenting during adult life most frequently represents cases either of re-coarctation following previous transcatheter or surgical therapy, or missed cases of native coarctation [3].

Nowadays, percutaneous stenting is an accepted form of treatment for isolated coarctation of the aorta [4]. Balloon expandable endovascular stents have been used in various locations since the 1980 [5]. Stents support the integrity of the vessel wall after balloon dilation by opposing the recoil the elastic vascular stenosis and re-adhering the torn intima to the media. This minimises the extension of wall tears and subsequent dissection or aneurysm formation [5].

Diagnosis and evaluation of coarctation is of great importance, not only before stenting but also after implantation, in order to assess the occurrence of restenosis. With regard to evaluation of aortic coarctation, cardiovascular magnetic resonance (CMR) imaging is the procedure of choice 17 . However; its use may be limited because of lack of availability or clinical contraindications.

On one hand, echocardiography is the only available bedside diagnostic tool. It can be used in the initial assessment and follow up after the intervention of patients with coarctation. Two- dimensional and Doppler techniques including analysis of pulse-wave and continuous-wave Doppler across the coarctation site and at the abdominal aorta are also used for the evaluation of coarctation [4].

The aim of this work is to evaluate the changes in a complete list of echocardiographic profiles in patients with aortic coarctation before and after stenting, and to determine the diagnostic value of these indices as an indicator of success of stenting.

\section{Patients and Methods}

\section{Inclusion criteria}

A prospective study included 40 consecutive patients, with a definite diagnosis of primary unoperated aortic coarctation based on clinical, Echocardiographic study, and confirmed by angiography, referred to the Pediatric Echo department of the National Heart Institute for further assessment according to the following protocol, a free written informed consent from all patients and their parents was taken.

\section{Exclusion criteria}

1. Patients with other concomitant lesions, including aortic stenosis or regurgitation, patent ductus arteriosus or ventricular septal defects.

2. Anomalies of the head and neck vessels.

3. Hypoplastic arch or evidence of sever collaterals during aortography.

The patients included in this study were subjected to the following:

a) Clinical assessment: i. History taking (personal, history of present illness, past history).

ii. Clinical examination (general, local).

b) Echo-Doppler study:

i. Echocardiographic assessment with our indices enrolled in this study was done twice for all patients, 24 hours before and 24 hours after stenting.

ii. Doppler study was performed using a Vivid 3 Imaging System (GE, USA) by one Echocardiologist before and after stenting who was totally blinded from the aim of this study.

iii. Descending aorta was evaluated during Doppler echocardiography from the standard suprasternal position with continuous-wave Doppler at coarctation site. Flow pattern of the abdominal aorta was assessed by continous wave Doppler obtained from subcostal view.

All studies, including pre- and post- stenting profiles, were performed with simultaneous electrocardiographic monitoring (ECG based), and the systole was assumed from the beginning of QRS till the end of T wave, onset of diastole was assumed at the end of the T wave till the end of PR segment.

Echocardiographic indices performed are defined as follows:

a. Deceleration time (DT): Measured from peak E velocity to the point where the slope of the slowing flow would intercept the baseline.

b. Systolic acceleration time: measured from the onset of the systolic upstroke to the systolic peak.

c. Pressure half-time (PHT) is the time interval for the peak pressure gradient to be reduced by one half $(\mathrm{PHT}=0.29 \times$ DT) [6].

d. Abdominal aortic pulse delay index is quantified by measuring the time to peak velocity in the abdominal aorta and comparing it with the same value measured from flow at the aortic annulus. This value should be indexed to the heart rate by dividing the absolute value by the square root of the RR interval [6].

e. Pulsatility index is the systolic velocity minus diastolic velocity divided by the mean (systolic velocity- diastolic velocity) / mean velocity [2]

f. Early diastolic velocity (EDV) is maximum diastolic velocity in early diastole [7].

g. Late diastolic velocity (LDV) is maximum diastolic velocity in late diastole (atrial contraction, calculated from end of $p$ wave on gated ECG) [8].

h. Peak systolic velocity is maximum systolic velocity [9].

i. Velocity time integral is the area under the velocity curve [9]. 


\section{Journal of Cardiology \& Cardiovascular Therapy}

j. Time to peak systolic velocity is the time from onset of the QRS complex to peak systolic velocity measured by pulsed wave Doppler echocardiography [7].

c) Cardiac catheterization: During diagnostic catheterization and during angioplasty for aortic coarctation these data were obtained: i. Site, length and width of coarctation segment.

ii. The characteristics of stenting, including length and width of stent, length and width of balloon, and before- and after-stent peak gradient of the catheter were recorded for all patients (Figure 1 \& 2) [10].

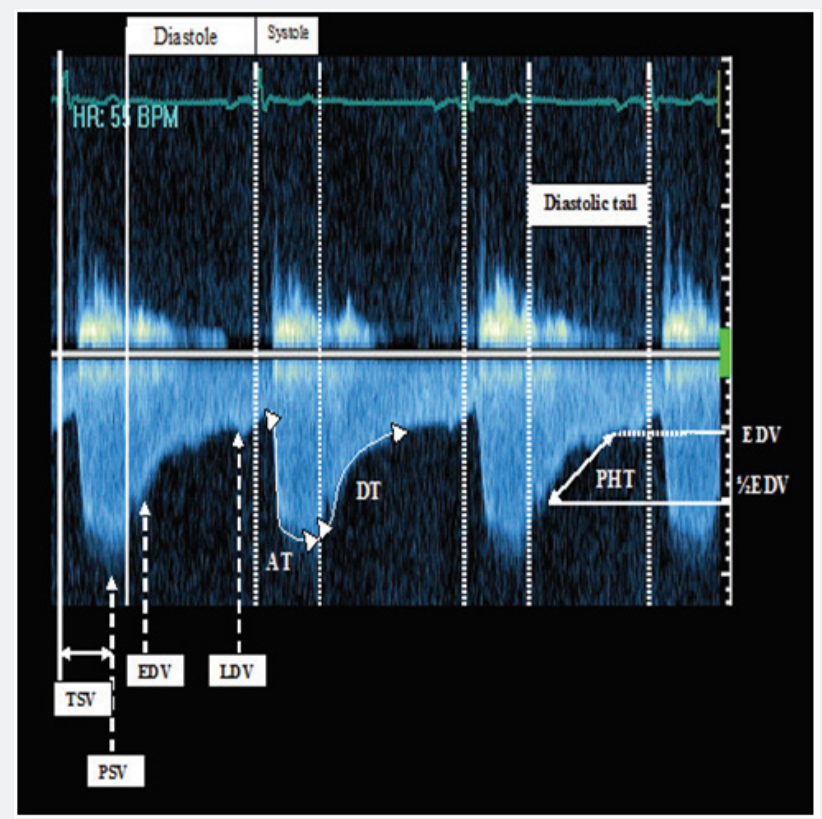

Figure 1: Continuous- wave Doppler echocardiography of descending aorta. AT: systolic Acceleration Time; DT: Deceleration Time; EDV: Early Diastolic Velocity; LDV: Late Diastolic Velocity; PHT: Pressure Half-Time (of diastole); PSV: Peak Systolic Velocity; TSV: Time to peak Systolic Velocity Wyse et al. [13].

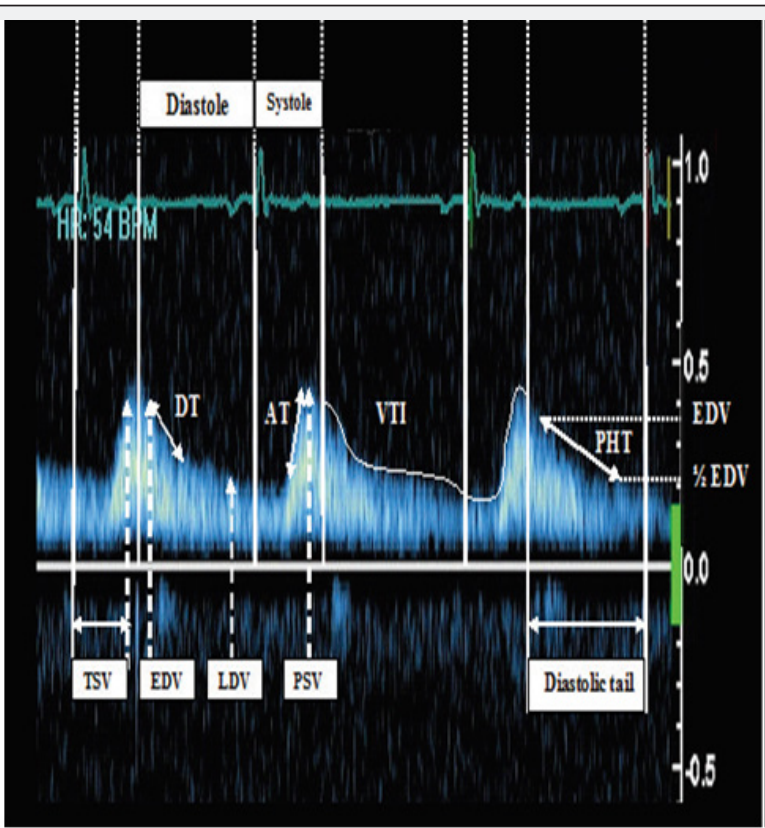

Figure 2: Continuous-wave Doppler echocardiography of the abdominal acceleration time is aorta. AT: systolic measured from the onset of the systolic upstroke to the systolic peak; DT: Deceleration Time is measured from Peak E velocity to the point where the slope of the slowing flow would intercept the baseline; EDV: Early Diastolic Velocity: maximum diastolic velocity on early diastole; LDV: Late Diastolic Velocity: maximum diastolic velocity on late diastole; PHT: Pressure Half- Time (of diastole) is the time interval for the peak diastolic pressure gradient to be reduced by one half; PSV: Peak Systolic Velocity: maximum systolic velocity; TSV: Time to peak Systolic Velocity: time beginning from onset of QRS complex to peak systolic velocity; VTI: Velocity Time Integral: the area under the curve, shown in both systole and diastole Wyse et al. [13]. 


\section{Statistical analysis:}

Data were entered checked and analyzed using Epi-Info version 6 and SPP for Windows version 8. Data were summarized using the arithmetic mean, the standard deviation, student $t$ test and chi-squared test.

Receiver operating characteristic curve (ROC) analysis was performed to assess the predictability of significant coarctation (pre-stenting condition) with the quantitative indices of the study, and then to compare area under the curve (AUC) of these variables. For this purpose, the first measured profiles before stenting were considered to be the values of patients with significant aortic coarctation, while the next measured indices after stenting were taken as the profiles of the individuals without coarctation. The cut-off points were then determined in each ROC analysis. The best predictive cut-off value was the one that gave the highest sensitivity and specificity simultaneously. The diagnostic values of each cut-off point, including sensitivity and specificity, were calculated and reported.

For all above mentioned statistical tests done, the threshold of significance is fixed at $5 \%$ level ( $p$-value).

Table 1: Demoghraphic and stenting characteristics of the patients.
The results were considered:

a. Significant when the probability of error is less than $5 \%$ $(\mathrm{p}<0.05)$.

b. Non-significant when the probability of error is more than $5 \%(p>0.05)$.

c. Highly significant when the probability of error is less than $0.1 \%(\mathrm{p}<0.001)$.

The smaller the p-value obtained, the more significant are the results.

\section{Results}

This prospective study enrolled 40 patients, with diagnosed coarctation of aorta), 32 males and 8 females with a mean age of 15.14 \pm 9.17 years (range 6.5-28) were enrolled in this study, the mean length of the stenosis was $18.5+9.5 \mathrm{~mm}$. In addition, the mean baseline ejection fraction was $58.55 \pm 5.10 \%$ (range $40-60 \%$ ) and 36 of patients were hypertensive. All baseline and stenting characteristics of the patients are listed in Table 1.

\begin{tabular}{|c|c|c|}
\hline \multicolumn{2}{|c|}{ Patients' Age (years) } & Patients' Age (years) $15.14 \pm 10.17$ \\
\hline \multicolumn{2}{|c|}{ Patients' gender } & \multirow{3}{*}{$\begin{array}{c}32 \\
8\end{array}$} \\
\hline \multicolumn{2}{|c|}{ Male } & \\
\hline \multicolumn{2}{|c|}{ Female } & \\
\hline \multicolumn{2}{|c|}{ Blood pressure } & \multirow{3}{*}{$\begin{array}{c}156 \pm 28 \\
93 \pm 16\end{array}$} \\
\hline \multicolumn{2}{|c|}{ Systolic blood pressure $(\mathrm{mmHg})$} & \\
\hline \multicolumn{2}{|c|}{ Diastolic blood pressure (mmHg) } & \\
\hline \multicolumn{2}{|c|}{ Aortic valve (\%) } & \multirow{3}{*}{$\begin{array}{l}26(65) \% \\
14(35) \%\end{array}$} \\
\hline \multicolumn{2}{|c|}{ BAV } & \\
\hline \multicolumn{2}{|c|}{ TAV } & \\
\hline \multicolumn{2}{|c|}{ Length of the stenosis (mm) } & $18.5+\_9.5$ \\
\hline \multirow{8}{*}{ Stenting data } & Length of the balloon (mm) & $20 \pm 8$ \\
\hline & Width of the balloon (mm) & $11+\_6$ \\
\hline & length of the stent (mm) & $28+66$ \\
\hline & width of the stent (mm) & $16+4$ \\
\hline & $\begin{array}{l}\text { Peak before-stenting gradient of the catheter } \\
\text { (mmHg) }\end{array}$ & $65+\_17$ \\
\hline & $\begin{array}{l}\text { Peak after-stenting gradient of the catheter } \\
\qquad(\mathrm{mm} \mathrm{Hg})\end{array}$ & $13+66$ \\
\hline & Before-stenting gradient of the catheter (\%) & $100 \%$ \\
\hline & After-stenting gradient of the catheter (\%) & $0 \%$ \\
\hline
\end{tabular}

All values for continuous variables are mean $\pm S D$.

Regarding symptoms and signs of presentation in our cases, 40 cases was symptomatic, 2 cases (5\%) complained mainly from growth failure and dysphagia with feeding, 38 cases (95\%) complained of intermittent claudications and coldness of lower limbs, 26 complained of exertional dyspnea (65\%), by physical examination; ejection systolic murmur over apex was found in 34 cases (85\%), brachio-femoral delay and blood pressure discrepancy between both arms was found in 38 cases (95\%), details of symptoms and signs in our cases are listed in Table 2. 
Table 2: Demoghraphic and stenting characteristics of the patients.

\begin{tabular}{|c|c|c|}
\hline & $\mathrm{N}=\mathbf{4 0}$ & $\%$ \\
\hline Asymptomatic & 0 & $0 \%$ \\
\hline Dysphagia, feeding problems or growth failure & 2 & $5 \%$ \\
\hline Intermittent claudication, coldness in lower limbs & 38 & $95 \%$ \\
\hline Ejection systolic murmur over apex, base or left interscapular region & 34 & $85 \%$ \\
\hline Blood pressure, pulse discrepancy, or brachio-femoral delay & 38 & $95 \%$ \\
\hline Exertional dyspnea & 26 & $65 \%$ \\
\hline
\end{tabular}

Table 3: Doppler Echocardiographic profile of abdominal and descending aorta before and after stenting (in values and percentage of change positive values show reduction whereas negative show increase in values after stenting.

\begin{tabular}{|c|c|c|c|c|c|c|c|c|}
\hline \multirow[b]{2}{*}{$\begin{array}{c}\text { Doppler } \\
\text { Echocardiographic } \\
\text { Profile }\end{array}$} & \multicolumn{2}{|c|}{$\begin{array}{l}\text { Mean Values before } \\
\text { Stenting }\end{array}$} & \multicolumn{2}{|c|}{ Mean Values after Stenting } & \multicolumn{2}{|c|}{$\begin{array}{l}\text { Mean Percentage Change } \\
\text { after Stenting (\%) }\end{array}$} & \multicolumn{2}{|c|}{$\begin{array}{l}\text { Mean Percentage Change } \\
\text { after Stenting (\%) }\end{array}$} \\
\hline & $\begin{array}{l}\text { Abdominal } \\
\text { Aorta }\end{array}$ & $\begin{array}{l}\text { Descending } \\
\text { Aorta }\end{array}$ & $\begin{array}{l}\text { Abdominal } \\
\text { Aorta }\end{array}$ & $\begin{array}{l}\text { Descending } \\
\text { Aorta }\end{array}$ & $\begin{array}{l}\text { Abdominal } \\
\text { Aorta }\end{array}$ & $P$ value & $\begin{array}{l}\text { Descending } \\
\text { Aorta }\end{array}$ & $P$ value \\
\hline PSV (m/s) & $0.64 \pm 0.75$ & $2.95 \pm 0.4$ & $0.81 \pm 0.16$ & $1.55 \pm 0.45$ & $-28.10 \pm 16.3$ & $<0.001$ & $95.88 \pm 35.4$ & $<0.001$ \\
\hline $\mathrm{EDV}(\mathrm{m} / \mathrm{s})$ & $0.57 \pm 0.24$ & $2.15 \pm 0.35$ & $0.36 \pm 0.18$ & $1.15 \pm 0.6$ & $33.25 \pm 18$ & $<0.001$ & $105.04 \pm 34.5$ & $<0.001$ \\
\hline $\mathrm{LDV}(\mathrm{m} / \mathrm{s})$ & $0.45 \pm 0.12$ & $0.7 \pm 0.55$ & $0.19 \pm 0.07$ & $0.42 \pm 0.15$ & $32.4 \pm 16$ & 0.002 & $40.1 \pm 22$ & $<0.001$ \\
\hline $\operatorname{AT}(\mathrm{m} / \mathrm{s})$ & $246 . \pm 45$ & $127 \pm 26$ & $120 \pm 24$ & $80 \pm 35$ & $55 \pm 17$ & $<0.001$ & $38 \pm 18$ & 0.020 \\
\hline PHT (m/s) & $215 \pm 35$ & $175 \pm 35$ & $93.00 \pm 16$ & $88.2 \pm 23$ & $3.51 \pm 23$ & $<0.001$ & $65 \pm 26$ & $<0.001$ \\
\hline Mean velocity (m/s) & $0.55 \pm 0.40$ & $1.96 \pm 0.35$ & $0.45 \pm 0.15$ & $1.25 \pm 0.4$ & $16 \pm 75.7$ & $<0.001$ & $0.75 \pm 0.28$ & $<0.001$ \\
\hline Mean PG & $0.65 \pm 0.25$ & $31 \pm 9$ & $0.75 \pm 0.35$ & $11 \pm 6$ & $64.95 \pm 16$ & 0.742 & $24.7 \pm 13$ & $<0.001$ \\
\hline $\mathrm{D} / \mathrm{S}$ ratio velocity & $0.93 \pm 0.21$ & $0.81 \pm 0.17$ & $0.43 \pm 0.27$ & $0.62 \pm 0.18$ & $16.4 \pm 11.5$ & $<0.001$ & $38.54 \pm 14$ & $<0.001$ \\
\hline VTI & $54.5 \pm 11.5$ & $173.5 \pm 45$ & $34.9 \pm 11.4$ & $88.5 \pm 22.5$ & $22.5 \pm 13$ & 0.005 & $88.5 \pm 22$ & $<0.001$ \\
\hline $\begin{array}{l}\text { Time to peak systolic } \\
\text { velocity }(\mathrm{m} / \mathrm{s})\end{array}$ & $315.8 \pm 46.40$ & $274 \pm 43.5$ & $248 \pm 47$ & $216.5 \pm 47$ & $34 \pm 14.40$ & $<0.001$ & $16.00 \pm 9.40$ & 0.026 \\
\hline Pulse delay & - & $7.3 \pm 4.8$ & - & $4.15 \pm 2.2$ & - & - & $61 \pm 24.5$ & $<0.001$ \\
\hline Pulsatility index & - & $0.9 \pm 0.35$ & - & $1.95 \pm 0.6$ & - & - & $-120.57 \pm 104$ & $<0.001$ \\
\hline
\end{tabular}

PSV: Peak Systolic Velocity; EDV: Early Diastolic Velocity; LDV: Late Diastolic Velocity; AT: Systolic Acceleration Time; PHT: Pressure Half-Time; PG: Peak Gradient; D/S: Diastolic Velocity/Systolic Velocity;

VTI: Velocity Time Integral

All $p$-values are from paired t-test and $p<0.05$ is considered significant.

The mean values of Doppler indices of the abdominal and descending aorta (before and after stenting) and mean percentages of changes after stenting are listed in Table 3. Stenting decreased the pulse-delay index from $7.3 \pm 4.8$ to $4.15 \pm 2.2$ ( $\mathrm{p}<0.001)$.

Additionally, significant reductions were noted in these indices:

1. Early diastolic velocity (EDV) ( $p$ value of early diastolic velocity-Abdomial aorta $<0.001$, $\mathrm{p}$ value EDV in descending aorta $<0.001)$.

2. Late diastolic velocity (LDV) (p value of LDV in abdominal aorta $=0.002$, $p$ value of LDV in descending aorta $<0.001$ ).

3. Acceleration time (AT) ( $p$ value of acceleration time in abdominal aorta $<0.001, \mathrm{p}$ value of acceleration time in descending aorta $=0.020$ ).

4. Pressure half time (PHT) ( $p$ value of PHT in abdominal aorta $<0.001$, $p$ value PHT in descending aorta $<0.001$ ).

5. Mean velocity ( $p$ value of mean velocity in abdominal aorta $<0.001$, p value of mean velocity in descending aorta $<0.001)$.

6. Mean PG ( $p$ value of mean pressure gradient in descending aorta $<0.001$ ).

7. Velocity time integral (VTI) ( $p$ value of VTI in abdominal aorta $=0.005$, p value of VTI in descending aorta $<0.001$ )

Time to peak systolic velocity was significantly increased after stenting ( $p$ value of time to peak systolic velocity in abdominal aorta $<0.001), \mathrm{p}$ value of time to peak systolic velocity in descending aorta $<0.001$ ). The largest percentage was achieved in the pulsatility index increased from $0.9 \pm 0.35$ to $1.95 \pm 0.6 \pm 0.51$, with percentage of increase (119.57\%), p value of pulsatility index of descending aorta $<0.001$ ).

The ROC curve analysis was performed to evaluate the diagnostic values of different Doppler echocardiographic indices 


\section{Journal of Cardiology \& Cardiovascular Therapy}

in order to differentiate significant coarctation (pre-stenting condition) from post-stenting conditions Diagnostic values of all 12 Doppler echocardiographic indices in both the abdominal and descending aorta are given in Table 4.

As shown in Table 4, except for the mean velocity and mean PG of the abdominal aorta, all other indices of the abdominal and descending aorta had a statistically significant area under the curve (AUC) to distinguish patients with significant aortic coarctation (pre-stenting condition) from post stenting condition.
The velocity time integral (VTI) of the descending aorta had the greatest AUC of 0.968 ( $p$ value of VTI in descending aorta <0.001) VTI had $95 \%$ sensitivity and $97.7 \%$ specificity for detection of significant aortic coarctation. A pulse delay of $>5.65$ had a sensitivity of $87 \%$ and specificity of $93.7 \%$ to diagnose significant aortic coarctation. Moreover, as illustrated in Table 4, a pulsatility index of $>1.23$ had $87 \%$ sensitivity and $91.7 \%$ specificity to differentiate significant coarctation (pre-stenting condition) from the poststenting condition.

Table 4: Diagnostic values of different cut-off points of Echocardiographic profiles of abdominal and descending aorta to differentiate significant coarctation (presenting condition) from poststenting condition (all data derived from ROC curve analysis).

\begin{tabular}{|c|c|c|c|c|c|}
\hline $\begin{array}{l}\text { Echocardiographic } \\
\text { Index }\end{array}$ & AUC & p-value & Cut-off Point & Sensitivity (\%) & Specificity (\%) \\
\hline \multicolumn{6}{|c|}{ Abdominal Aorta } \\
\hline $\mathrm{PSV}(\mathrm{m} / \mathrm{s})$ & 0.769 & $<0.001$ & 0.585 & 76.4 & 89 \\
\hline $\operatorname{EDV}(\mathrm{m} / \mathrm{s})$ & 0.922 & $<0.001$ & 0.305 & 81 & 63.5 \\
\hline $\mathrm{LDV}(\mathrm{m} / \mathrm{s})$ & 0.763 & 0.004 & 0.236 & 71.2 & 93 \\
\hline $\operatorname{AT}(\mathrm{m} / \mathrm{s})$ & 0.877 & $<0.001$ & 154.4 & 87.8 & 91.5 \\
\hline PHT (m/s) & 0.975 & $<0.001$ & 107 & 88 & 86 \\
\hline Mean velocity (m/s) & 0.192 & 0.145 & - & - & - \\
\hline Mean PG & 0.547 & 0.512 & - & - & - \\
\hline $\mathrm{D} / \mathrm{S}$ ratio velocity & 0.934 & $<0.001$ & 0.487 & 92 & 88 \\
\hline VTI & 0.769 & 0.003 & 24.5 & 77 & 72 \\
\hline $\begin{array}{l}\text { Time to peak systolic } \\
\text { velocity }(\mathrm{m} / \mathrm{s})\end{array}$ & 0.937 & $<0.001$ & 323.5 & 84 & 97 \\
\hline \multicolumn{6}{|c|}{ Descending Aorta } \\
\hline $\mathrm{PSV}(\mathrm{m} / \mathrm{s})$ & 0.914 & $<0.001$ & 3.4 & 87.7 & 91 \\
\hline $\mathrm{EDV}(\mathrm{m} / \mathrm{s})$ & 0.937 & $<0.001$ & 0.87 & 89 & 93 \\
\hline $\mathrm{LDV}(\mathrm{m} / \mathrm{s})$ & 0.945 & $<0.001$ & 0.405 & 89 & 87 \\
\hline $\operatorname{AT}(\mathrm{m} / \mathrm{s})$ & 0.712 & 0.029 & 81 & 73 & 68 \\
\hline PHT (m/s) & 0.957 & $<0.001$ & 88.6 & 84 & 89 \\
\hline Mean velocity (m/s) & 0.925 & 0.192 & 1.24 & 94 & 89 \\
\hline Mean PG & 0.523 & 0.123 & 14.5 & 89 & 92 \\
\hline $\mathrm{D} / \mathrm{S}$ ratio velocity & 0.916 & $<0.001$ & 0.38 & 88 & 91.5 \\
\hline VTI & 0.968 & $<0.001$ & 97 & 95 & 97.7 \\
\hline $\begin{array}{l}\text { Time to peak systolic } \\
\text { velocity }(\mathrm{m} / \mathrm{s})\end{array}$ & 0.718 & 0.027 & 271 & 63 & 90 \\
\hline Pulse delay & 0.914 & $<0.001$ & 6.2 & 87 & 93.7 \\
\hline Pulsatility index & 0.964 & $<0.001$ & 1.27 & 88 & 93 \\
\hline
\end{tabular}

PSV: Peak Systolic Velocity; EDV: Early Diastolic Velocity; LDV: Late Diastolic Velocity; AT: Systolic Acceleration Time; PHT: Pressure Half-Time; PG: Peak Gradient; D/S: Diastolic Velocity/Systolic Velocity; VTI: Velocity Time Integral; AUC: Area Under Curve.

Table 5: Evaluation of the correlation between severity of coarctation (baseline peak gradient of the catheter) and presenting Echocardiographic profiles of abdominal and descending aorta.

\begin{tabular}{|c|c|c|c|}
\hline Echocardiographic Index & Correlation Coefficient (R) & p-value \\
\hline \multicolumn{2}{|c|}{ Abdominal Aorta } \\
\hline PSV (m/s) & -0.076 & 0.625 & NS \\
\hline EDV (m/s) & 0.078 & 0.684 & NS \\
\hline LDV (m/s) & -0.023 & 0.878 & NS \\
\hline AT (m/s) & 0.017 & 0.938 & NS \\
\hline
\end{tabular}


Journal of Cardiology \& Cardiovascular Therapy

\begin{tabular}{|c|c|c|c|}
\hline PHT (m/s) & 0.587 & 0.001 & HS \\
\hline Mean velocity (m/s) & 0.059 & 0.715 & NS \\
\hline Mean PG & 0.109 & 0.57 & NS \\
\hline D/S ratio velocity & 0.056 & 0.825 & NS \\
\hline VTI & 0.395 & 0.0026 & NS \\
\hline Time to peak systolic velocity (m/s) & -0.009 & 0.787 & NS \\
\hline PSV (m/s) & \multicolumn{2}{|c|}{ Descending Aorta } & S \\
\hline EDV (m/s) & 0.241 & 0.232 & S \\
\hline LDV (m/s) & 0.446 & 0.027 & NS \\
\hline AT (m/s) & 0.567 & 0.005 & HS \\
\hline PHT (m/s) & 0.088 & 0.627 & HS \\
\hline Mean velocity (m/s) & 0.573 & 0.001 & S \\
\hline Mean PG & 0.452 & 0.026 & NS \\
\hline D/S ratio velocity & 0.414 & 0.042 & S \\
\hline VTI & 0.358 & 0.088 & NS \\
\hline Pulsatility index & 0.632 & 0.01 & NS \\
\hline
\end{tabular}

${ }^{*}$ Paired $t$ test

PSV: Peak Systolic Velocity; EDV: Early Diastolic Velocity; LDV: Late Diastolic Velocity; AT: Systolic Acceleration Time; PHT: Pressure Half-Time; PG: Peak Gradient; D/S: Diastolic Velocity/Systolic Velocity; VTI: Velocity Time Integral; AUC: Area Under Curve. All data derived from two-sided Pearson correlation analysis.

As shown in Table 5, the baseline peak aortic gradient measured by catheter was significantly correlated with some of the pre-stenting echocardiographic profiles of the abdominal and descending aorta, the strongest correlation of the peak gradient was observed with pressure half-time as a direct relationship ( $\mathrm{r}$ abdominal aorta $=0.573$, $\mathrm{p}$ value abdominal aorta $<0.001 ; \mathrm{r}$ descending aorta $=0.584, \mathrm{p}$ value descending aorta $<0.003$ ). The velocity-time integral of the descending aorta was also directly correlated with before-stenting peak gradients ( $\mathrm{r}$ descending aorta $=0.632$, $p$ value descending aorta $<0.010$ ). Other correlations are shown in Table 5. The possible correlation of the baseline peak aortic gradient with the mean percentage change in echocardiographic profiles after stenting was also evaluated. Our findings showed a reverse correlation between severity of coarctation and changes in LDV after stenting. The higher the gradient, the lower the change in the LDV of the abdominal aorta ( $r$ abdominal aorta $=-0.567, \mathrm{p}$ value of abdominal aorta $=0.033$ ). By contrast, changes in the PHT of the abdominal aorta were directly correlated with the baseline catheter gradient ( $\mathrm{r}$ abdominal aorta $=0.587, \mathrm{p}$ value abdominal aorta $=0.001$ ). Changes in the other echocardiographic indices were not significantly correlated with the baseline aortic gradient.

\section{Discussion}

Congenital heart disease (CHD) occurs in approximately 8 of every 1000 live births, half of whom require surgical or other forms of treatment. Coarctation of aorta is a complex congenital heart defects involving the outflow tracts of the heart, the aorta and its branches.

The embryological development of the heart and the great vessels is one same process that goes hand in hand and this means that the presence of an easily detected cardiac congenital abnormality does not mean the exclusion of associated extra cardiac vascular abnormality. It also means that the more the complex the cardiac abnormality, the more the complex the expected extra cardiac vascular abnormality could should be.

Major advancements in the surgical and medical management of cases with coarctation of aorta allowed a large percentage of these cases to survive to adulthood. This required proper diagnosis and timed management to provide the child with a good chance of leading normal life with minimal if any disability.

Cardiac imaging is a fundamental element of the discipline of pediatric cardiology, required at all stages of patient care. Imaging Provides a detailed depiction of the anatomy and physiology of congenital heart disease (CHD), can define management and monitor follow-up.

An accurate, 3D evaluation of the cardiac and related arterial anatomy is critical for the clinical management of pediatric patients with complex congenital heart disease. 3D imaging has the ability to demonstrate the shapes of, and spatial relationships between, the great arteries, proximal and distal branch pulmonary arteries, coronary vessels and anomalous pulmonary venous or systemic 
connections. Three-dimensional information about extra-cardiac morphological characteristics may determine subsequent surgical intervention, follow up the residuals of interventions, and assist with estimation of prognosis.

Recent advances in diagnostic radiology have radically altered the approach to the diagnosis of congenital heart disease (CHD). Sophisticated trans-thoracic and transesophageal echocardiography is perhaps the best-known example. Additionally, state of the art magnetic resonance (MR) and multislice computed tomography (CT) scanners, each with cardiac packages, can render diagnostic angiocardiography unnecessary at times. With an accurate diagnosis, interventional angiocardiography, embolotherapy, surgical correction, or even cardiac transplantation can be accomplished for palliation or cure.

In spite of these imaging breakthroughs, many if not most coarctation of aorta patients, independent of age, are referred to the radiologist with a request for a chest radiograph, either one or two view. Plain chest x-rays have long been established as a valuable tool in the evaluation of patients with heart murmur or the suspicion of CHD. It had been taught that coarctation of aorta have classic manifestations on plain chest x-ray, (Figure) for coarctation of aorta.

The advent of echocardiography (echo) in the 1970s led to a revolution in the non-invasive diagnosis of heart disease. Echo became the mainstay of diagnosis and follow-up for congenital heart disease, used to determine chamber pressures and function, detect intracardiac anomalies, fair data about extracardiac anatomy (pulmonary \& systemic vessels) prior to surgery (Oh et al. 2009).

In addition to echocardiographic 2-D imaging, the Doppler examination has become an essential component of the complete echocardiographic evaluation. The field of cardiac US continues to grow rapidly: recent clinical additions include 3-D imaging, harmonic imaging, and contrast echocardiography.

Echocardiography is a great modality for initial assessment because of its mobility and availability however; it may not be the perfect diagnostic tool because it is usually limited by the acoustic window, spatial resolution, and the subjective interpretation of the operator. An incorrect echo diagnosis might result in the wrong operation and the risk of avoidable mortality, a mistake that could potentially have been corrected by MSCT (Tsai et al. 2008).

Cardiac catheterization has traditionally been the procedure utilized to complement echo, providing hemodynamic information and enabling visualization of extracardiac great vessels. However, the role of diagnostic cardiac catheterization in pediatric cardiology is evolving for a number of reasons.

The major advantages of digital angiocardiography include the ability to eliminate bony structures by subtraction techniques thus reducing the risk of motion mis-registration artifacts and more importantly to use the sequential data to create quantitative analyses. One of the advantages of the technique is that it is less operator dependant, functional data like intra cardiac or intra vascular pressure gradients or blood oxygenations levels are obtainable [11].

On the other hand one of the potential negative repercussions of cardiac catheterization is the invasive nature of procedure (e.g. arterial and venous compromise, stroke, bleeding) and of the exposure to radiation. In many centres, cardiac catheterization is now reserved for patients in whom hemodynamic data is essential (e.g. unexplained pulmonary hypertension), and/or in whom interventional procedures are necessary.

Understanding the varied cardiac anatomy often requires additional imaging, as a supplement to more traditional first-line methods (i.e. echocardiography or cardiac catheterization). MRI and CT has rapidly established itself as a complementary modality to echocardiography in a wide variety of clinical scenarios where echo is either hindered by lack of acoustic windows, or is unable to provide all the necessary information for therapeutic decision making. It has also decreased the need for routine diagnostic cardiac catheterization prior to surgery.

Krishnamurthy reported that cardiovascular magnetic resonance (CMR) imaging plays an important role in the evaluation of patients with complex CHD. It overcomes many of the limitations of echocardiography (e.g., restricted acoustic windows), computed tomography (e.g., exposure to ionizing radiation, limited functional information), and cardiac catheterization (e.g., exposure to ionizing radiation, morbidity, high cost).

Coarctation of the aorta is characterised by anatomical obstruction in the descending aorta. It is difficult to evaluate this obstruction because of the variability in cardiac output, number and size of collaterals, and peripheral resistance. (Teien DE and Wendel H) Primary clinical diagnosis and subsequent assessment of the severity of coarctation and re-coarctation of the aorta have traditionally been made based on the judgment of the character of the femoral pulse. Also known as a secondary event, absent, weakened or delayed femoral pulses occur as a result of obstruction in aortic coarctation. The pressure drop across the obstruction (the gradient), pressure half-time, and diastolic flows are widely used but inaccurate indices to diagnose aortic coarctation. They can be affected by many other factors such as cardiac output, lesion length, the presence of collateral networks, and aortic compliance. (Tacy TA, Baba K) for this, CMR, $\mathrm{CT}$, traditional Echo-Doppler assessment of coarctation proved to be sufficient tools.

Stent implantation has been used as a reliable treatment for coarctation of the aorta. It has several advantages, rendering it superior to angioplasty alone. Bussadori $\mathrm{C}$ et al. The effect of stents on blood flow dynamics is not well known. Moreover, despite the importance of close follow up to evaluate complications and the long-term effect on the blood pressure of these patients, there 
are no adequate long-term follow-up indices for these patients. Therefore the present study was carried out to find reliable, quantitative Doppler echocardiographic indices for assessment of the severity of coarctation of the aorta before stenting and comparing these indices with the post-stenting condition. This would provide a valuable profile to indicate successful stent implantation.

All previous methods, including monitoring the blood pressure, two-dimensional echocardiography, cardiac magnetic resonance (CMR) and angiography have failed to give favourable results to rely on for early detection and follow up of restenosis, this is evidenced by persistent hypertension, even in the absence of a recurrent or residual stenosis, insufficient anatomical evaluation of two-dimensional echocardiography, and disrupted MRI by metallic artifacts (or noise) have limited the value of these indices to assess the patient at post-intervention follow up (Hamdan MA, Maheshwari S), Furthermore, angiography as an invasive procedure has known complications and should be restricted to use in cases planned only for reintervention.

Doppler echocardiography overcomes these problems in the follow up of such patients. However, echocardiography may be less sensitive than angiography, spiral computed tomography and MRI in detecting aneurysms after stent placement (Hamdan MA, Maheshwari S).

Based on our results, the Doppler echocardiographic profile was found to be valid for differentiating significant coarctation (presenting) from the successfully stented cases (after stenting), with high diagnostic values. As demonstrated in the results, continuous flow was significantly decreased from before to after stenting in both the descending and abdominal aorta. Moreover, monophasic systolic flow was shown to increase significantly after stenting.

In comparison with a few similar studies, Mivelaz \& Di Bernardo [3] we assessed more indices. According to our results, aortic pulse delay decreased after stenting. The results also showed that a pulsatility index of $<1.27$ was suggestive of significant coarctation of the aorta. This cut-off point was matching with cut-off point calculated by Shokoufeh Hajsadeghi et al. which was $<1.2$, and mismatching with cut-off value of $<2$ in a study by Silvilairat et al. Hagler DJ et al. Currently, it is known that obstructed blood flow due to aortic coarctation leads to pressure drop and loss of the pulse wave distal to the stenosis. This can be observed by echocardiography typically as decreased pulsatility of the abdominal aorta after cardiac systole Pfammatter JP, Stocker FP.

Early and late diastolic velocities were found to be significant markers in the assessment of the severity of coarctation. In addition, mean gradient of the descending aorta was significantly reduced by as much as $65 \%$ following stenting, this was matching with Shokoufeh Hajsadeghi et al. Which reported decrease in mean gradient in descending aorta $58 \%$ following stenting, the residual gradient could be the result of changed flow dynamics along the stent Tan et al. [12].

However, in some patients, there was an under- or overestimation of the pressure gradient across the coarctation site on Doppler echocardiography. As mentioned, these are affected by other factors, such as cardiac output, lesion length, the presence of collateral networks, and aortic compliance. Therefore pressure gradient alone as an index of aortic narrowing is often inadequate.

Although the mean velocity in both the descending and abdominal aorta significantly decreased after stenting, the difference was more significant in the descending aorta, with an approximately $60 \%$ reduction. This was matching with Shokoufeh Hajsadeghi et al. Similarly, the acceleration time in the descending aorta was different from the corresponding measurement in the ascending aorta in coarctation. Shaddy RE, Snider AR. This is manifested clinically by radial femoral delay and diminished pulses distal to the coarctation. After stent implantation, the acceleration time showed statistically significant decreases in both the descending and abdominal aorta.

Based on our findings, the velocity-time integral and time to peak systolic velocity can be also used as new markers of significant coarctation. Both indices significantly decreased after stenting. We also found pressure half-time indices (systolic and diastolic velocity half-times, systolic and diastolic pressure halftimes) can be used to assess the severity of coarctation, with sensitivities of 88 and $84 \%$ and specificities of 86 and $89 \%$ for the abdominal aorta and descending aorta, respectively. This is minimally different from the results of Shokoufeh Hajsadeghi et al. who reported sensitivities of 87 and $81.8 \%$ and specificities of 100 and $87 \%$ for the abdominal aorta and descending aorta, respectively.

These findings were in keeping with the results of previous investigations by Carvalho et al. and Tan et al. [12] who reported a significant effect of coarctation of the aorta on these indices.

A study by Lim and Ralston however was in disagreement with regard to systolic indices. According to Lim, Diastolic velocities (DVs) and diastolic pressure decays have been shown to provide invaluable information for assessing the severity of coarctation Hoadley SD, Duster MC.

The index of D/S ratio velocity was first used by Tan et al. [12] as a marker of significant coarctation. They demonstrated that a D/S ratio velocity of $>0.53$ had a sensitivity of $100 \%$ and specificity of $96 \%$ for detecting significant aortic coarctation. They believed that by correlating diastolic with systolic velocity, this ratio would be less affected by variations in heart rate, stroke volume, systemic blood pressure and aortic compliance. Tan et al. [12] in our study we found cut-off value of $>0.38$ for $\mathrm{D} / \mathrm{S}$ ratio in descending aorta to has a sensitivity of $88 \%$, specificity of $91.5 \%$ for detecting significant coarctation which was close to $\mathrm{D} / \mathrm{S}$ ratio reported by Tan et al. [12] (0.53), Shokoufeh Hajsadeghi et al. ( 0.43 value showed sensitivity $95.7 \%$, specificity $87 \%)$. 
Whereas the ratio of $>0.487$ in the abdominal aorta had a sensitivity of $92 \%$ and specificity of $88 \%$ in defining significant coarctation of the aorta. This is close to results of Shokoufeh Hajsadeghi et al. which reported that ratio of $>0.43$ in the abdominal aorta had a sensitivity of $81.8 \%$ and specificity of $91.3 \%$ in defining significant coarctation of the aorta.

Besides evaluation of the diagnostic value of the echocardiographic indices, a correlation analysis was also performed in our study to assess the relationship between the severity of coarctation before stenting and the echocardiographic indices. As shown, PHT and VTI of the abdominal aorta and EDV, EDV, PHT, mean velocity and mean peak gradient of the descending aorta correlated significantly with the peak gradient in the coarctation site, measured by catheterisation prior to stent implantation [13-15].

In addition, the higher pre-stenting gradients were associated with lower changes in LDV of the abdominal aorta, while changes in PHT of the abdominal aorta were directly correlated with the baseline gradient. The observed correlation between the baseline severity of coarctation and the changes in PHT after stenting leads us to conclude that this index (PHT) is probably the best to determine stenting outcome and the probable occurrence of restenosis. Nevertheless, it should be evaluated in further studies.

The results of the present study showed that a complete set of Doppler echocardiographic profiles could potentially provide a valid method to detect success stenting of significant aortic coarctation. Velocity-time integral, time to peak systolic velocity, systolic acceleration time and mean velocity were sensitive and specific enough to detect significant aortic coarctation, as were peak systolic, early diastolic and late diastolic velocities, pressure half-time, peak gradient and $\mathrm{D} / \mathrm{S}$ ratio velocities, which were validated in previous studies.

To the best of our knowledge this is the second evaluation of such a complete list of Doppler echocardiographic indices to detect significant coarctation of the aorta. Our findings emphasise the advantages of Doppler echocardiography for close monitoring of patients with aortic coarctation. Although these echocardiographic indices do improve dramatically in patients who underwent stenting, they never return to normal values even if no residual significant stenosis exists (peak pressure gradient by catheter less than $20 \mathrm{mmHg}$ after stenting means no significant residual stenosis).

\section{Conclusion}

We found a significant difference between pre- and post-stenting echocardiographic values, Post-stenting echocardiographic profiles of each patient could therefore provide an individualised and reliable reference value of his/her normal aortic haemodynamics, and early detection of restenosis could be achieved by comparison of post-stenting values with follow-up values, to our knowledge; there is no follow up study used these parameters for follow up of patients with coarctation after stenting, to prove the significance of these parameters to detect restenosis, however we recommend more studies to use these parameters for follow up to clarify their significance to detect restenosis compared to golden standard (cardiac magnetic resonance imaging or multi-slice computed tomography.

\section{References}

1. Anagnostopoulos-Tzifa A (2007) Management of aortic coarctation in adults: endovascular versus surgical therapy. Hellenic J Cardiol 48(5): 290-295.

2. Gosling RG, King DH (1947) Arterial assessment by Doppler shift ultrasound. Proc R Soc Med 67(6 Pt 1): 447-449.

3. Mivelaz Y, Di Bernardo S, Meijboom EJ, Sekarski N (2008) Validation of two echocardiographic indexes to improve the diagnosis of complex coarctations. Eur J Cardiothorac Surg 34(5): 1051-1056.

4. Mühler EG, Neuerburg JM, Rüben A, Grabitz RG, Günther RW, et al. (1993) Evaluation of aortic coarctation after surgical repair: role of magnetic resonance imaging and Doppler ultrasound. Br Heart J 70(3): 285-290.

5. Ovaert C, Benson LN, Nykanen D, Freedom RM (1998) Transcatheter treatment of coarctation of the aorta: a review. Pediatr Cardiol 19(1): $27-44$.

6. Oh JK, Sweard JB, Tajik AJ (2007) The Echo Manual. ( $3^{\text {rd }}$ edn), Lippincott Williams \& Wilkins, Philadepphia, USA.

7. Gardner BI, Bingham SE, Allen MR, Blatter DD, Anderson JL (2009) Cardiac magnetic resonance versus transthoracic echocardiography for the assessment of cardiac volumes and regional function after myocardial infarction: an intra subject comparison using simultaneous intra subject recordings. Cardiovasc Ultrasound 7: 38.

8. Smiseth OA, Tendera M (2008) Diastolic heart failure. (1 $1^{\text {st }}$ edn), Springer, London, UK.

9. Goldberg BB, Merton DA, Deane CR (1997) An atlas of ultrasound color flow imaging. ( $1^{\text {st }}$ edn), Taylor \& Francis, London, USA.

10. Wyse RK, Robinson PJ, Deanfield JE, Tunstall Pedoe DS, Macartney FJ (1999) Use of continuous wave Doppler ultrasound velocimetry to assess the severity of coarctation of the aorta by measurement of aortic flow velocities. Br Heart J 52(3): 278-283.

11. Bailliard F, Hughes ML, Taylor AM (2008) Introduction to cardiac imaging in infants and children: Techniques, potential, and role in the imaging work-up of various cardiac malformations and other pediatric heart conditions. Eur J Radiol 68(2): 191-198.

12. Tan JL, Babu-Narayan SV, Henein MY, Mullen M, Li W (2005) Doppler echocardiographic profile and indexes in the evaluation of aortic coarctation in patients before and after stenting. J Am Coll Cardiol 46(6): 1045-1053.

13. Chalil S, Stegemann B, Muhyaldeen S, Khadjooi K, Smith RE, et al. (2007) Intraventricular dyssynchrony predicts mortality and morbidity after cardiac resynchronization therapy: study using cardiovascular magnetic resonance tissue synchronization imaging. J Am Coll Cardiol 50(3): 243-252.

14. Suárez de Lezo J, Pan M, Romero M, Medina A, Segura J, et al. (1999) Immediate and follow-up findings after stent treatment for severe coarctation of aorta. Am J Cardiol 83(3): 400-406.

15. Suárez de Lezo J, Pan M, Romero M, Medina A, Segura J, et al. (1995) Balloon-expandable stent repair of severe coarctation of the aorta. Am Heart J 129(5): 1002-1008. 
(C) This work is licensed under Creative

DOI: 10.19080/JOCCT.2018.11.555808
Your next submission with Juniper Publishers will reach you the below assets

- Quality Editorial service

- Swift Peer Review

- Reprints availability

- E-prints Service

- Manuscript Podcast for convenient understanding

- Global attainment for your research

- Manuscript accessibility in different formats

( Pdf, E-pub, Full Text, Audio)

- Unceasing customer service

Track the below URL for one-step submission https://juniperpublishers.com/online-submission.php 